\title{
WEB ALERT
}

Editor Chin Lee, MD, MPH

Northwestern University, Feinberg School of Medicine, Division of

Rheumatology, 240 E. Huron Street, McGaw Pavilion, Suite M-300,

Chicago, IL 60611, USA.

E-mail: c-lee17@northwestern.edu

\section{National Osteoporosis Society}

http://www.nos.org.uk

The National Osteoporosis Society (NOS) is a national charity based in the United Kingdom, dedicated to improving the prevention, diagnosis, and treatment of fragile bone disease. The website provides introductory information on risk factors, preventive measures, diagnosis, and treatments related to osteoporosis. Information on these topics is even available as free PDF files in the Punjabi and Welsh languages. Visitors can purchase booklets and video and audio material online. Interested individuals can join the NOS and receive professional publications that include "Osteoporosis News" and "Osteoporosis Review," which provide the latest news and information on osteoporosis. Visitors can register to join the NOS discussion forum at no cost. Patients should find the NOS website particularly useful.

\section{Osteoporosis Australia}

http://www.osteoporosis.org.au

Osteoporosis Australia promotes awareness, education, prevention, management, research, and advocacy related to osteoporosis. Visitors can access educational materials related to all aspects of osteoporosis as downloadable PDF files. Well-written educational material focused on the risk factors, identification, management, and prevention of osteoporosis are available for consumers, general practitioners, and pharmacists. Handouts providing visitors with introductory materials are featured in several languages (Arabic, Chinese, English, Greek, Italian, and Vietnamese). Downloadable publications on osteonecrosis of the jaw and osteoporosis in men may be of particular interest to both patients and health care professionals.

\section{Osteoporosis Canada}

http://www.osteoporosis.ca

Osteoporosis Canada is a national organization that works to educate, empower, and support individuals and communities in the prevention and treatment of osteoporosis. Accordingly, the group's website offers pragmatic information for patients, their families, and health care professionals on pertinent topics related to osteoporosis. An archive of personal stories illustrates how individuals with diverse backgrounds have handled osteoporosis. Additionally, the website offers information on existing osteoporosis support groups throughout Canada. A "News" section, aimed at informing both health care professionals and nonprofessionals, covers relevant topics on all aspects of osteoporosis, and "In the News" links directly to recent media coverage. Visitors can also estimate their level of calcium intake by accessing the "Calcium Calculator," can take an online osteoporosis quiz and learn more about related information on nutrition and physical activity, and can even obtain calcium-rich recipes. There are links to other sites that visitors may find useful. The website is also available in French. 